EPJ Web of Conferences 44, 01002 (2013)

DOI: $10.1051 /$ epjconf/20134401002

C) Owned by the authors, published by EDP Sciences, 2013

\title{
Evidence of a characteristic ignition length of a flame
}

\author{
Y.Baara, K.Khalloufi, and N.Zekri* \\ Université des Sciences et de la Technologie d'Oran Mohamed Boudiaf, Département de Physique, \\ LEPM, BP 1505 El Mnaouer, Oran, Algerie
}

\begin{abstract}
Ignition process of vegetation from a cylindrical flame radiation is examined using Koo and Pagni [1] model. The radiation flux is power-law decreasing with distance [2], while the ignition time increases exponentially. This last behavior yields a characteristic length of fuel ignition from a flame, inducing a percolation type phase transition.
\end{abstract}

\section{1 introduction}

Percolation theory is one of the simplest statistical physics models describing propagation in disordered media [3]. Percolation models are now applied to several fields of physics ranging from electronic and optical properties of composite materials to epidemics via forest fires $[4,5]$.

A finite propagation/non-propagation threshold transition is induced by a finite characteristic or path length [6]. A finite path length means an exponentially decreasing connection probability with the distance between nodes. In the case of human epidemics and forest fires, the corresponding small world networks exhibit long-range connections [7] with an exponentially decreasing connectivity distribution leading to a finite percolation threshold. In other epidemic networks such as computer networks called scale free networks, there is no percolation threshold because the connectivity distribution is power-law decreasing even if the path length remains finite [7]. Obviously forest fires exhibit a finite percolation threshold as fire can either spread or not depending on the vegetation density, terrain roughness and/or weather conditions. On the other hand, in the absence of wind, flame radiation is the main origin fire propagation. However, following Billaud et al. [2] and Mudan [8], the radiation flux is asymptotically power-law decreasing with distance, and thus the characteristic length of flame radiation diverges. Fire propagates by igniting the connected sites not only by heating them. Ignition is a complex process. It includes water evaporation and pyrolysis processes. Is there a characteristic length induced by ignition process? The answer of this question is the aim of this paper.

\section{Ignition process}

Let us consider the influence of a flame on a combustible located at a distance $y$ from the flame. A simple physical model of Koo et al. [1], based on energy conservation and detailed heat transfer mechanisms, is used for steady-state contiguous fire spread in a thermally-thin uniform porous fuel bed (Fig.1).

*nzekri@yahoo.com

This is an Open Access article distributed under the terms of the Creative Commons Attribution License 2.0, which permits unrestricted use, distribution, and reproduction in any medium, provided the original work is properly cited. 


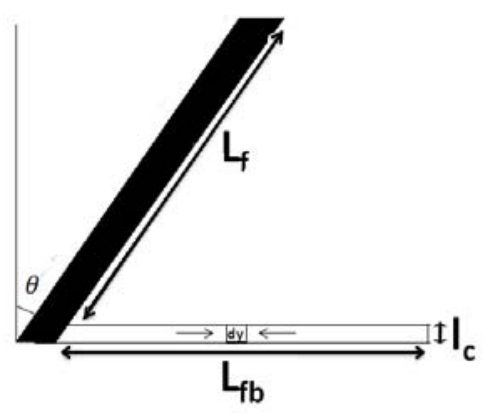

Fig. 1.Flame spread schematic.

In this model, the fuel bed has a length $L_{f b}$ and is assumed to be a thin, one-dimensional, homogenous, porous fuel layer (its thickness $l_{c}$ is small compared to the fuel bed and flame sizes). There is thus no temperature difference between top and bottom surfaces at the same distance from the flame. The emitted radiations from the flame contribute to heating the surface of the combustible layer. The flame is assumed to be line fire; it is in fact a three-dimensional sheet with a cylindrical shape of length $L_{f}$, but its radius $r$ is used only for flame emissivity calculations from its surface. Combustion and chemical reactions are thus assumed to be infinitely fast. The reference system is attached to the flame sheet so that the flame is fixed at $y=0$. The tilt angle of the flame $\theta$ is caused by the wind speed $\mathrm{U}_{\mathrm{w}}$ and the gravitational constant $g$. Itis approximated as [1]

$$
\theta=\tan ^{-1}\left[\frac{1.4 U_{W}}{\sqrt{g L_{f}}}\right]
$$

Thermal heat supplied by the flame radiations leads to the fuel layer degradation and to rise the temperature until it reaches the ignition temperature $T_{i g n}$. The temperature of the combustible layer is thus time and distance dependent $\left(\mathrm{T}_{\mathrm{y}}(\mathrm{t})\right)$. The combustible layer is at room temperature $T_{r t}$ for $y=\infty$, and at the flame temperature $\mathrm{T}_{\mathrm{f}}$ for $y=0$.

Consider a volume element $d y \times l_{c} \times l($ lbeing the width of the fuel bed)of the combustible layer heated by a flame radiations at a distance $y$. The total flux $q_{T}$ received by this element is

$$
\mathrm{q}_{\mathrm{T}}=\mathrm{q}_{\mathrm{sr}}+\mathrm{q}_{\mathrm{rl}}+\mathrm{q}_{\mathrm{sch}}+\mathrm{q}_{\mathrm{scb}}
$$

In the present work the wind speed is neglected, and the fuel bed is flat. The tilt angle is thus neglected as well as the surface heading $\left(\mathrm{q}_{\mathrm{sch}}\right)$ and backing $\left(\mathrm{q}_{\mathrm{scb}}\right)$ convection. The radiation flux $\mathrm{q}_{\mathrm{sr}}$ received from the flame is given by:

$$
\mathrm{q}_{\mathrm{sr}}=\frac{\mathrm{a}_{\mathrm{fb}} \times \sigma \times \varepsilon_{\mathrm{f} \times} T_{f}^{4}}{\mathrm{l}_{\mathrm{c}}} \mathrm{F}
$$

Where $a_{f b}$ denotes the absorption coefficient of the combustible element layer, $T_{f}$ and $\varepsilon_{\mathrm{f}}$ are respectively the flame emissivity and temperature, $\sigma$ the Stephan Bolzmann constant and $F$ is the view factor, i.e. the proportion of the total energy emitted by the flame that reaches the receiving surface element. This factor was be determined by Monte-Carlo simulations for a cylindrical flame shape [2]. The Monte-Carlo method used reduces to a stochastic ray-tracing method where discrete energy bundles are sent through the computational domain with the ray direction being sampled.

The total emission of an isothermal area element $d A_{i}$ depends on the emissiondirection (i.e. the polar $\varphi$ and the azimuthal angle $\psi$ ), generated by using the random numbers $0 \leq R_{\varphi} \leq 1$ and $0 \leq R_{\psi} \leq 1$

$$
\varphi=\sin ^{-1}\left(\sqrt{R_{\varphi}}\right)
$$




$$
\psi=2 \pi R_{\psi}
$$

The radiative heat flux received by any target surface element $d A_{j}$ depends on the number of quantum $N_{i j}$ as

$$
\mathrm{q}_{\mathrm{j}}=\frac{\mathrm{W}}{\mathrm{dA}_{\mathrm{j}}} \sum_{i=1}^{I} N_{i j}
$$

Where $\mathrm{W}$ is the amount of the radiative energy carried by each quantum.
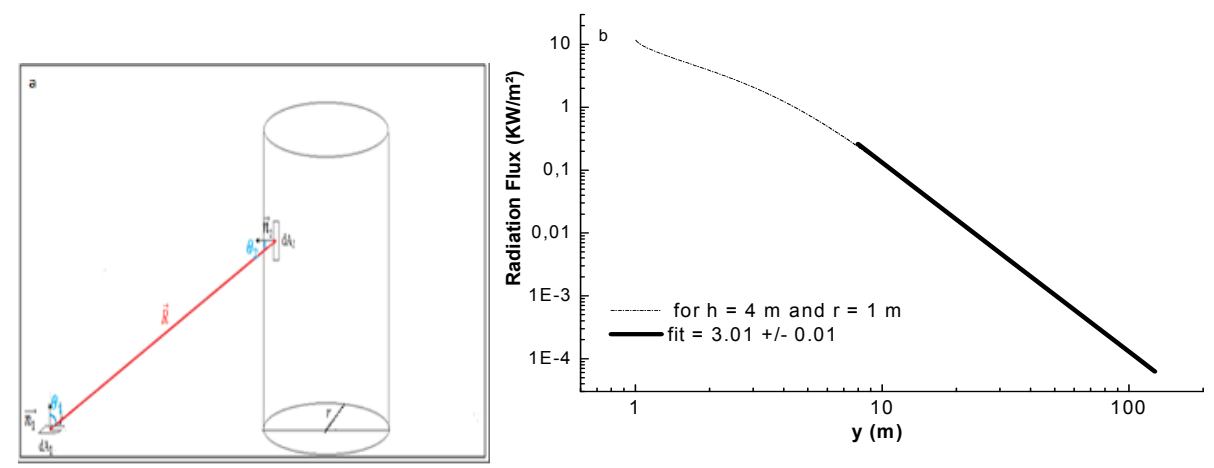

Fig.2.a) Schematic representation of the physical model, b) Radiative heat flux received by a horizontal target surface and vertical flame (flame emissive power $25 \mathrm{KW} / \mathrm{m} 2$ ).

The flux is power-law decreasing with the distance to the flame. From Fig.2, the radiation interaction has thus a diverging characteristic length. This is obvious as sun radiations influence objects on the earth whose distance to the sun can be considered as infinite, but they are not ignited. Therefore, ignition process should have a characteristic length induced by an exponential behaviour.

The radiation loss of the surface element $\mathrm{q}_{\mathrm{rl}}$ is given by

$$
\mathrm{q}_{\mathrm{rl}}=-\frac{\sigma \times \varepsilon_{\mathrm{c}} \times\left(T_{y}^{4}-T_{r t}^{4}\right)}{\mathrm{l}_{\mathrm{c}}}
$$

Where $\varepsilon_{\mathrm{c}}$ denotesthe emissivity of the combustible element layer.

At $373 \mathrm{~K}^{\circ}$ the total flux $q_{T}$ is used for water evaporation. Above this critical temperature, the total flux increases the temperature of the combustible element until it reaches the ignition temperature so that

$$
\mathrm{q}_{\mathrm{T}}=\left\{\begin{array}{c}
\rho_{c} C_{p c} \emptyset \frac{d T}{d t}, \text { for } T \neq 373 K^{\circ} \\
-\rho_{c} h_{v a p} \varnothing \frac{d W}{d t}, \text { for } T=373 K^{\circ}
\end{array}\right.
$$

Where $\rho_{c}$ is the fuel density, $C_{p c}$ its specific heat, $\varnothing$ the volume fraction of the solid phase (fine particles of vegetation), $W$ is the water content, and $h_{v a p}$ the latent heat of water evaporation. To solve Eq.8, the following boundary conditions on temperature and water content are needed

$$
\left\{\begin{array}{cc}
T(0)=T_{\text {ign }} ; & T(\infty)=T_{r t} \\
W(0)=0 ; & W(\infty)=W_{0}
\end{array}\right.
$$

With $\mathrm{W}_{0}$ is the initial moisture content of the fuel bed. A first order approximation is used iteratively until reaching the ignition temperature. The results are in agreement with the fourth order Runge Kutta method. 


\section{Results}

In this section, we consider a cylindrical flame with a height $h=4 \mathrm{~m}$ and a radius $r=1 \mathrm{~m}$. Fig.3 shows the time dependence of the temperature of fuel elements located at various positions $y$. When temperature reaches the ignition temperature (here about $580^{\circ}$ ), the fuel element is ignited. Before ignition, three different behaviors appear; water heating phase, the evaporation phase and the pyrolysis phase. A plateau appears at $373^{\circ} \mathrm{K}$ due to the latent energy at the water evaporation phase. Below this plateau, temperature increases exponentially with time, while it increases as a power-law above the plateau. This increase becomes slower as the distance $y$ to the flame increases. A nonlinear increase of the ignition time with $y$ is observed in this figure.

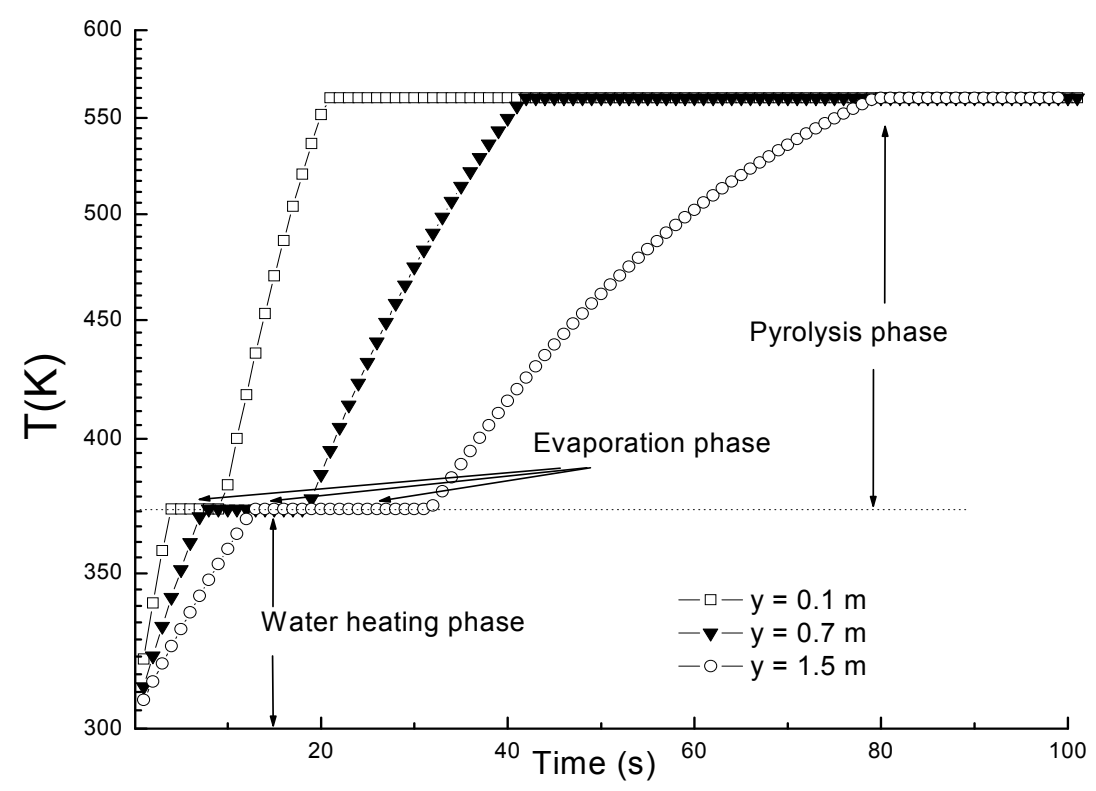

Fig. 3. Time evolution of the fuel temperature for various distances $y$.

The ignition time dependence on the distance $y$ is shown in Fig.4 (semi-log plot). For small distances (about $0.5 \mathrm{~m}$ ), the ignition time is power-law increasing with the distance. This increase becomes exponential above this distance, yielding a characteristique length $l_{c}$ (here $l_{c}=2.77 \pm 0.01$ )

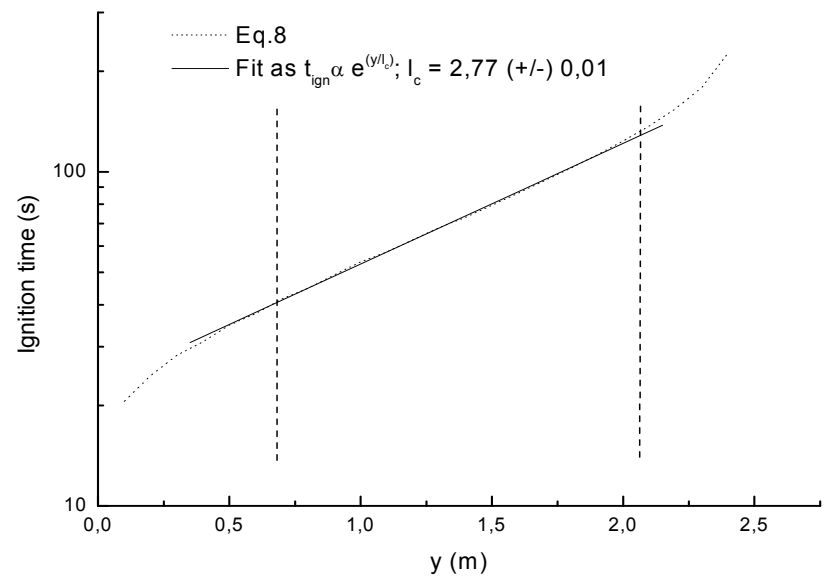

Fig.4.Ignition time as a function of $y$.for a flame length $L_{f}=4 \mathrm{~m}$ and a radius $r=1 \mathrm{~m}$ 


$$
t_{\text {ign }} \propto \exp ^{\frac{y}{l_{c}}}
$$

The ignition time increase becomes faster than the exponential above this length. This means that above this distance, the fuel bed elements cannot be ignited. Obviously this length allows us to simulate fire propagation by using a small deterministic area of radius $l_{c}$ (called the influence zone), instead of the whole system, which is highly computer time consuming. This length was introduced previously to study forest fire spread and percolation [5].

\section{Conclusions}

Using a simple physical model of heat transfer that includes radiation processes of a cylindrical flame, the existence of a characteristic length of ignition was demonstrated. This length, which is the cause of the propagation/ non propagation phase transition, was deduced from the exponential increase of the ignition time with the distance to the flame. A further investigation is in course where the characteristic length is determined as a function of the wind speed, the fuel bed slope and its moisture content.

\section{References}

1. E.Koo, P.J.Pagni, J.Woycheese, S.Stephens, D.Weise, and J.Hu, A Simple Physical Model for Forest Fire Spread Rate, Fire Safety Science-Proceedingsofthe Eighth International Symposium, International Association for Fire Safety Science, London, UK, 2005 pp. 851862.

2. Y.Billau,A.Kaiss, J.-L.Consalvi and B.Porterie, Int.J.Therm.Science, 50, 2 (2011).

3. D. Stauffer and A. Aharony, Introduction to Percolation Theory (Taylor and Francis, London, 1992).

4. Clerc J. P. Giraud G. Laugier J. M. and Luck J. M. 1990 adv. Phys. 39,191

5. $\quad$ N. Zekri, B. Porterie, J.P. Clerc, and J.C. Loraud, Phys.Rev.E 71, 046121 (2005).

6. G. Grimmett, Percolation (Springer-Verlag, Heidelberg, 1999); H.E. Stanley, Introduction to Phase Transitions and Critical Phenomena (Oxford University Press, London, 1971).

7. R.Albert and A.-L.Barabasi, Rev.Mod.Phys. 74, 47 (2002).

8. K. Mudan, 10, 59 (1984).

9. J.R. Howell, Thermal Radiation Heat Transfer, fourth ed. (Taylor and Francis,New York, 2001);See also: http://www.me.utexas.edu/whowell/. 